$>$ Les cellules souches embryonnaires sont communément associées à la thérapie cellulaire. Elles présentent toutefois en parallèle un tout autre intérêt pour la santé, comme outil à usage multiple de la recherche pharmacologique. Les cellules souches embryonnaires possèdent en effet, pour des études in vitro, l'avantage d'être une ressource biologique inépuisable du fait de leur immortalité, ce qui permet de s'appuyer sur le même fond génétique dans des études successives. Elles sont également la seule source connue à ce jour de cellules cultivables présentant le phénotype de n'importe quel lignage à n'importe quel stade de différenciation. Même si l'exploitation industrielle de ces deux propriétés dépend encore en partie d'innovations technologiques qui restent à venir, les travaux utilisant ce nouvel outil plein de promesses en recherche pharmacologique sont bel et bien en marche. <

\section{Les cellules souches \\ embryonnaires et la pharmacologie}

Delphine Laustriat, Jacqueline Gide, Céline Héchard, Marc Peschanski

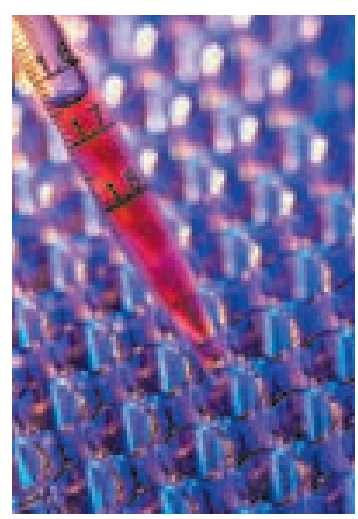

Inserm/UEVE U 861, I-STEM, AFM, Genopole Campus 1, 5 , rue Henri Desbruères, 91030 Évry Cedex, France. dlaustriat@istem.genethon.fr mpeschanski@ istem.genethon.fr www.istem.eu

biologiques des cellules qu'elles sont censées modéliser. Leurs capacités prolifératives, qui font en grande partie leur intérêt, deviennent un handicap difficile à surmonter, lorsque la cible des effets recherchés est présente dans des cellules post-mitotiques. Les cellules dérivées de prélèvement possèdent les avantages et inconvénients inverses. Dans tous les cas, les phénotypes cellulaires disponibles sont limités par les capacités propres de la ressource cellulaire utilisée, dont la capacité de différenciation est au mieux multipotente. L'arrivée «sur le marché » des cellules souches embryonnaires (CSE), de souris d'abord puis humaines (CSEh) (Figure 1) [1, 2], a de ce fait été le signal d'une activité intense - au moins de réflexion visant à établir les conditions d'une substitution des modèles cellulaires anciens par ceux dérivés de cette ressource nouvelle [3-8].

\section{Quel(s) rôle(s) pour les cellules souches embryonnaires dans l'identification} de cibles thérapeutiques et de composés actifs?

Une première exploitation des CSEh découle de leur capacité à donner accès à des mécanismes moléculaires et fonctionnels dans un environnement biologique humain et phénotypiquement adapté. Cette capacité peut se décliner de deux façons, selon que l'on fait appel à un modèle physiologique ou pathologique pour l'identification de cibles thérapeutiques et/ou 
de composés pharmacologiques actifs. Les lignées de CSEh natives constituent un modèle physiologique permettant de rechercher l'effet de molécules sur des structures subcellulaires ou des systèmes de signalisation intracellulaire en vue d'une modification d'intérêt thérapeutique. La «manifestation» d'une pathologie peut, quant à elle, être induite dans ces CSEh natives après transfection d'une construction génique connue pour ses effets pathologiques $[9,10](\rightarrow)$. Les lignées de CSch établies à partir d'embryons porteurs d'un gène muté condui-

$(\rightarrow) \mathrm{m} / \mathrm{s} 2008$, $n^{\circ} 4$, p. 419 sent, en outre, à des modèles exprimant spontanément des anomalies moléculaires [11-14]. Ces lignées, actuellement au nombre d'une trentaine, ne sont disponibles que pour les maladies dépistées par diagnostic pré-implantatoire. L'arrivée récente des cellules iPS humaines (induced pluripotent stem cells) [1517] $(\rightarrow)$ devrait permettre d'élargir le champ de ces modèles spontanés. Les iPS sont des cellules « repro-

$(\rightarrow) \mathrm{m} / \mathrm{s} 2008$, $n^{\circ} 1$, p. 102 grammées » par surexpression de facteurs de transcription, conduisant à un phénotype présentant vraisemblablement des propriétés similaires aux CSEh. En partant de cellules de patients, il devrait ainsi être possible d'accéder à des modèles «spontanés» pour toutes les pathologies et de bénéficier de modèles correspondant à différents stades d'évolution de la maladie.

La maladie de Huntington est l'exemple d'une pathologie pour laquelle le potentiel de ces différents types d'approches de modélisation est évalué (Figure 2). Comme le montre la stratégie de la société de biotechnologie Dialectica $[18,19]$, les modèles issus de CSEh seront vraisemblablement dans un premier temps destinés à une utilisation à petite échelle pour la validation de cibles thérapeutiques et l'optimisation de composés actifs. Une fois les contraintes technologiques d'une utilisation à grande échelle maîtrisées (voir plus loin), cette nouvelle génération de modèles pourra également montrer son intérêt dès les étapes précoces de criblage de vastes chimiothèques (ressources pharmacologiques et génomiques) pour l'identification et non plus seulement la confirmation de cibles et de molécules actives [3].

\section{Cellules souches embryonnaires et toxicologie prédictive}

Outils potentiels de modélisation pathologique, les cellules souches embryonnaires et leurs dérivés sont également envisagés aujourd'hui comme des modèles pertinents de la physiologie cellulaire avec, en vue, une exploitation intense dans le domaine de la toxicologie prédictive, préoccupation essentielle des industries pharmaceutique et cosmétique.

\section{L’EST : application logique}

pour un modèle embryonnaire

$\varepsilon n$ tout premier lieu, les cellules souches embryonnaires sont un excellent modèle de repro-toxicologie grâce à... ce qu'elles sont, à savoir une phase très précoce de l'ontogenèse. Cet intérêt a été reconnu depuis longtemps, notamment par l'ECVAM (European centre for the validation of alternative methods) qui, dans les années 1990, a mis au point l'EST (embryonic stem cell test). Ce test utilise des CSE murines (CSEm) pour évaluer le potentiel cytotoxique d'une molécule, ainsi que son effet sur la différenciation [20]. Pour cela, des CSEm sont mises en contact avec le composé à tester puis placées dans des conditions de différenciation spontanée. Une partie d'entre elles évolue vers un phénotype de cardiomyocytes contractiles, ce qui permet de déterminer I'ID50, concentration en composé qui inhibe de $50 \%$ la différenciation spontanée des cellules CSEm en cellules contractiles. Ce paramètre étant difficilement mesurable, il tend à être remplacé par la quantification de l'expression de marqueurs de différenciation. La cytotoxicité, quant à elle, est évaluée en déterminant I'IC50, concentration

\begin{tabular}{|c|c|c|c|}
\hline & Cellules immortalisées & Cellules primaires & CSEh et dérivés \\
\hline Origine & $\begin{array}{c}\text { Cellules tumorales ou cellules } \\
\text { primaires immortalisées }\end{array}$ & Prélèvement & Blastocyste \\
\hline Types cellulaires disponibles & Dépend du prélèvement & Dépend du prélèvement & Tous \\
\hline Quantité disponible & Illimitée & $\begin{array}{l}\text { Limitée, } \\
\text { dépend du prélèvement }\end{array}$ & Illimitée \\
\hline Prolifération & +++ & - & +++ \\
\hline Stabilité génétique & - & +++ & +++ \\
\hline \multirow{2}{*}{ Autres remarques } & Immortalisation & \multirow{2}{*}{$\begin{array}{l}\text { Dédifférenciation, } \\
\text { variations inter-donneurs }\end{array}$} & $\begin{array}{l}\text { Dans certains cas, lignées } \\
\text { modèles spontanés de maladie }\end{array}$ \\
\hline & Modifications métaboliques & & $\begin{array}{l}\text { Unique modèle pour étudier } \\
\text { l'état indifférencié }\end{array}$ \\
\hline
\end{tabular}

Tableau I. Comparaison des principaux modèles cellulaires utilisés dans le développement du médicament. 


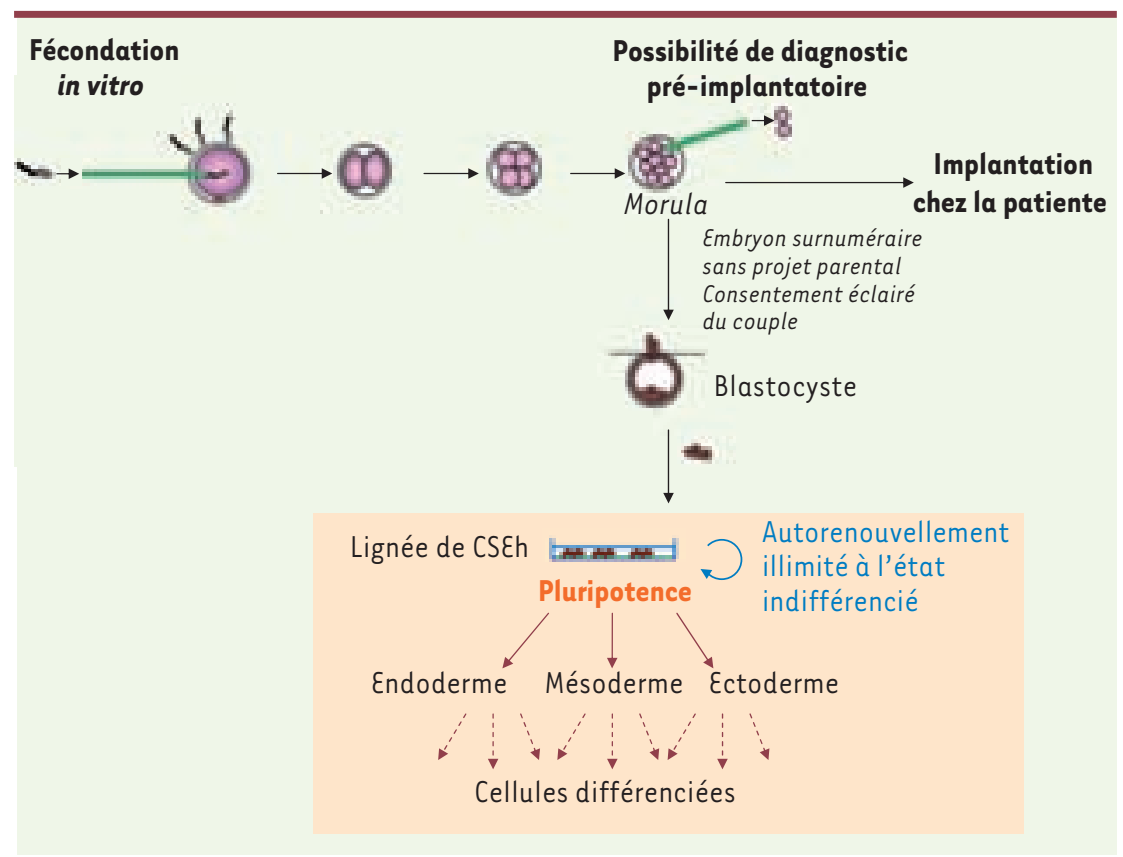

Figure 1. Dérivation et propriétés biologiques des CS\&h. Les CS\&h ont été mises en évidence en 1998 par l'équipe de Thomson [1]. En France, leur utilisation à des fins de recherche est autorisée par la loi de Bioéthique de 2004 et encadrée par l'Agence de la biomédecine. Les lignées de CSEh sont établies à partir d'embryons surnuméraires issus de fécondation in vitro qui ne font plus l'objet d'un projet parental. Au stade de développement précoce «blastocyste », qui correspond aux jours $5 \frac{1}{2}$ à $71 \frac{1}{2}$ après fécondation chez l'homme, la masse cellulaire interne est prélevée et mise en culture sur une couche de cellules nourricières. Les CSEh se développent sur ce tapis cellulaire et forment des colonies. Les couples présentant des antécédents familiaux pour certaines pathologies transmissibles génétiquement ont la possibilité d'effectuer un diagnostic préimplantatoire. Pour cela, au stade «morula » où

coexistent 8 cellules, 2 d'entre elles sont prélevées afin d'effectuer le dépistage. De la même manière que pour les embryons sains, les blastocystes dont le génome présente la mutation associée à une maladie et qui ne font plus l'objet de projet parental pourront conduire à l'établissement de lignées de CSEh. Dans ce cas précis, les lignées sont des modèles spontanés de la pathologie. Les lignées de CSદh obtenues, qu’elles portent ou non la mutation associée à une maladie, se caractérisent par une capacité d'autorenouvellement illimitée à l'état indifférencié. Elles sont, en outre, pluripotentes, c'est-à-dire capables, par différenciation, de générer tous les types cellulaires présents dans un organisme, à l'exception des annexes embryonnaires (Figure adaptée d'après [35]).

en composé actif inhibant de moitié la croissance des CSEm par rapport à des cellules d'une lignée adulte contrôle. Sur 20 produits toxiques caractérisés in vivo, ce test a permis d'en classer correctement $78 \%$ au sein des catégories suivantes: composé non/faiblement/ fortement toxique [21]. Etant donné les différences inter-espèces, notamment en terme de métabolisme - comme illustré par le thalidomide et l'acide 13 -cis-rétinoïque, sévères tératogènes chez l'homme n'entraînant pas d'effet particulier sur des modèles murins [22] - plusieurs équipes de recherche se sont attachées ces dernières années à développer des tests comparables à partir des CSEh (Figure 3). C'est notamment le cas des équipes de l'ECVAM et des sociétés de biotechnologie ProteoSys et Cellartis, associées dans le projet européen ReProTect (http://www.reprotect.eu/) [23]. Ce programme regroupe 35 partenaires européens (académiques, industries pharmaceutiques, entreprises de biotechnologies...), dans l'objectif de développer une séquence de tests in vitro et in silico pour évaluer la toxicité de composés sur toutes les étapes de la reproduction et du développement en vue de réduire, remplacer ou du moins réajuster le recours à l'expérimentation animale. Les premiers résultats de la transposition de I'EST aux CSEh ont été publiés récemment par Adler et al. [22], qui ont validé l'effet cytotoxique du 5-fluorouracile et de l'acide transrétinoïque. Concernant l'évaluation de l'effet des molécules sur la différenciation cardiaque, une première phase de leur travail a consisté à adapter la séquence des marqueurs à tester.
En effet, la cinétique de la cardiogenèse de même que les acteurs impliqués diffèrent entre lignées murines et humaines. Afin de ne pas seulement détecter les composés interférant avec la différenciation cardiaque, le programme Reprotect a également pour objectif d'étendre I'EST à la différenciation neurale.

\section{Métabolisme et toxicité :} autres applications classique mais néanmoins essentielles pour les dérivés de CSEh Ensuite, et d'une façon beaucoup plus générale, la diversité des phénotypes cellulaires humains auxquels les CSEh permettent d'accéder offre potentiellement à la recherche pharmacologique l'accès à des populations prioritaires dans la recherche toxicologique tels que les hépatocytes ou les cardiomyocytes - types cellulaires pour lesquels il n'existe jusqu'ici pas de modèle satisfaisant conjointement des exigences qualitatives et quantitatives. Le gouvernement et l'Association britannique des industries pharmaceutiques (ABPI - Association of the British Pharmaceutical Industry, équivalent du LદEM français - Les Entreprises du Médicament) ont considéré la question comme si importante qu'ils en ont fait un thème prioritaire ces dernières années. Une phase 
de réflexion, engagée par le travail d'une commission réunissant les meilleurs spécialistes académiques et industriels, a conduit à un long rapport opérationnel (http://www.sc4sm.org/downloads/SC4PTREPORT.pdf), base fondatrice de leur action. Roche, Astra Zeneca et
Glaxo SmithKline se sont ensuite associés à des laboratoires académiques et des sociétés de biotechnologie pour mener à bien le programme dit SC4SM (stem cells for safer medicine). Ce projet a pour but de développer

\section{A Approches de modélisations possibles}

\begin{tabular}{|c|c|c|c|}
\hline \multicolumn{1}{|c|}{} & \multicolumn{2}{|c|}{$\begin{array}{c}\text { Modèles pour l'identification de cibles thérapeutiques } \\
\text { et/ou de composés pharmacologiques actifs }\end{array}$} \\
\cline { 2 - 4 } & Modèles natifs & \multicolumn{2}{|c|}{ Modèles de pathologie } \\
\cline { 2 - 4 } $\begin{array}{c}\text { Exemples appliqués } \\
\text { à la maladie } \\
\text { de Huntington }\end{array}$ & $\begin{array}{c}\text { Modèle permettant } \\
\text { d'étudier l'interaction } \\
\text { entre REST/NRSF } \\
\text { et le site REl/NRSE }\end{array}$ & $\begin{array}{c}\text { Modèles obtenus par } \\
\text { modification génétique } \\
\text { Introduction } \\
\text { de la mutation huntingtine, } \\
\text { mimer un gain } \\
\text { ou une perte de fonction } \\
\text { décrit dans la maladie }\end{array}$ & Lignées issues du DPI, iPS \\
\hline
\end{tabular}

B Système Dia-NRSELUC

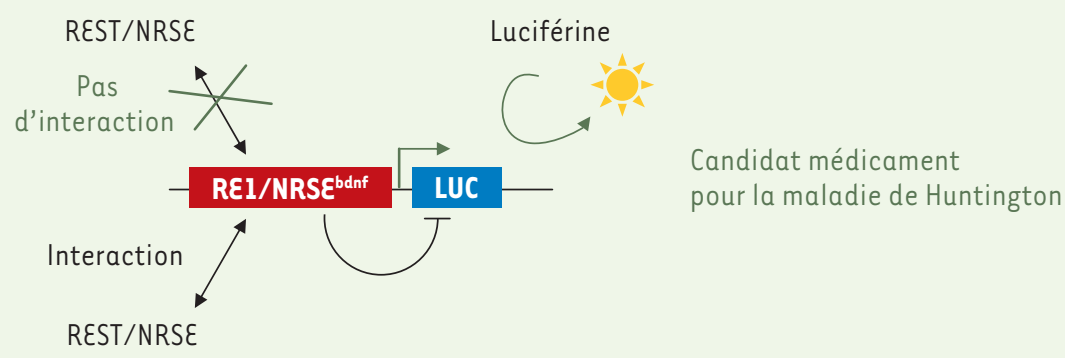

\section{Stratégie de Dialectica pour l'identification de composés actifs} pour la maladie de Huntington

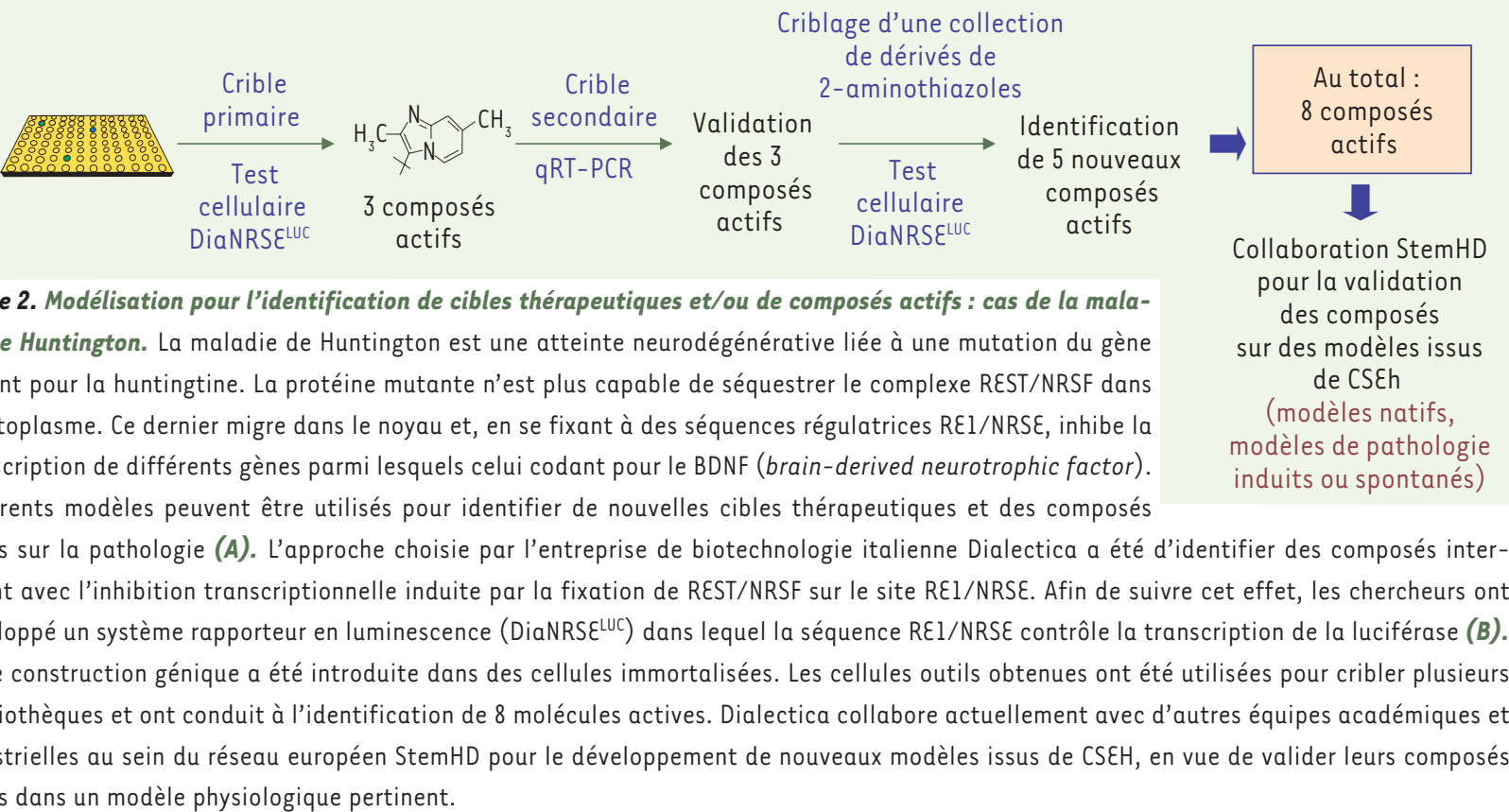


les outils nécessaires pour l'utilisation des cellules souches dans les tests de sûreté des médicaments au cours des étapes précoces de leur développement. De nombreux types cellulaires issus de CSEh pourront trouver ici un intérêt, afin de fournir une carte d'identité des effets de candidats médicaments sur les différents tissus de l'organisme. SC4SM se focalise à l'heure actuelle sur les hépatocytes et devrait, si l'intérêt des CSEh est démontré, élargir son champ d'investigation aux cardiomyocytes, autre type cellulaire essentiel dans les études de toxicité des médicaments (Figure 3).

Le choix des hépatocytes s'explique aisément par leur rôle central dans le métabolisme. Ils permettent d'étudier les enzymes impliquées dans la transformation d'un candidat médicament, d'identifier ses métabolites et d'anticiper des interactions médicamenteuses. Toute toxicité sur ces cellules implique un danger et doit impérativement être également détectée. Les modèles actuellement utilisés sont des hépatocytes primaires ou des lignées immortalisées (lignées HepG2, HepaRG) qui présentent des limites respectivement en termes d'approvisionnement et de fidélité des réactions métaboliques par rapport aux hépatocytes humains dans leur contexte physiologique [24]. La recherche concernant la différenciation des CSEh en hépatocytes a considérablement progressé ces dernières années. Il est désormais possible d'obtenir des cellules «hepatocyte-like » en une vingtaine de jours, en mettant des CSEh en culture dans des conditions induisant successivement une activation endodermique puis une maturation en hépatocytes. Le rendement en hépatocytes, qui était de $2 \%$ en 2003 , atteint désormais $70 \%$ dans deux protocoles publiés ces derniers mois [25, 26]. Les cellules obtenues par ces protocoles expriment un certain nombre de marqueurs caractéristiques des hépatocytes. D'un point de vue fonctionnel, ces cellules «hepatocyte-like » sont capables de synthétiser et de sécréter l'albumine in vitro et de stocker du glycogène; elles expriment une glycoprotéine $P$ fonctionnelle. Pour ce qui est du métabolisme, elles expriment des enzymes impliquées dans les réactions de phase I (oxydation des molécules les rendant plus hydrophiles pour mieux les éliminer), mais également de phase II (augmentation

\section{Toxicité développementale}

Perturbations des mécanismes de différenciation? Effet sur la prolifération?
Stade de différenciation des CSEh

Indifférencié

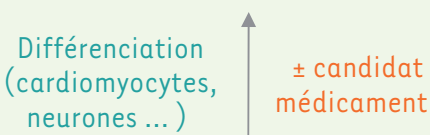

neurones...) médicament

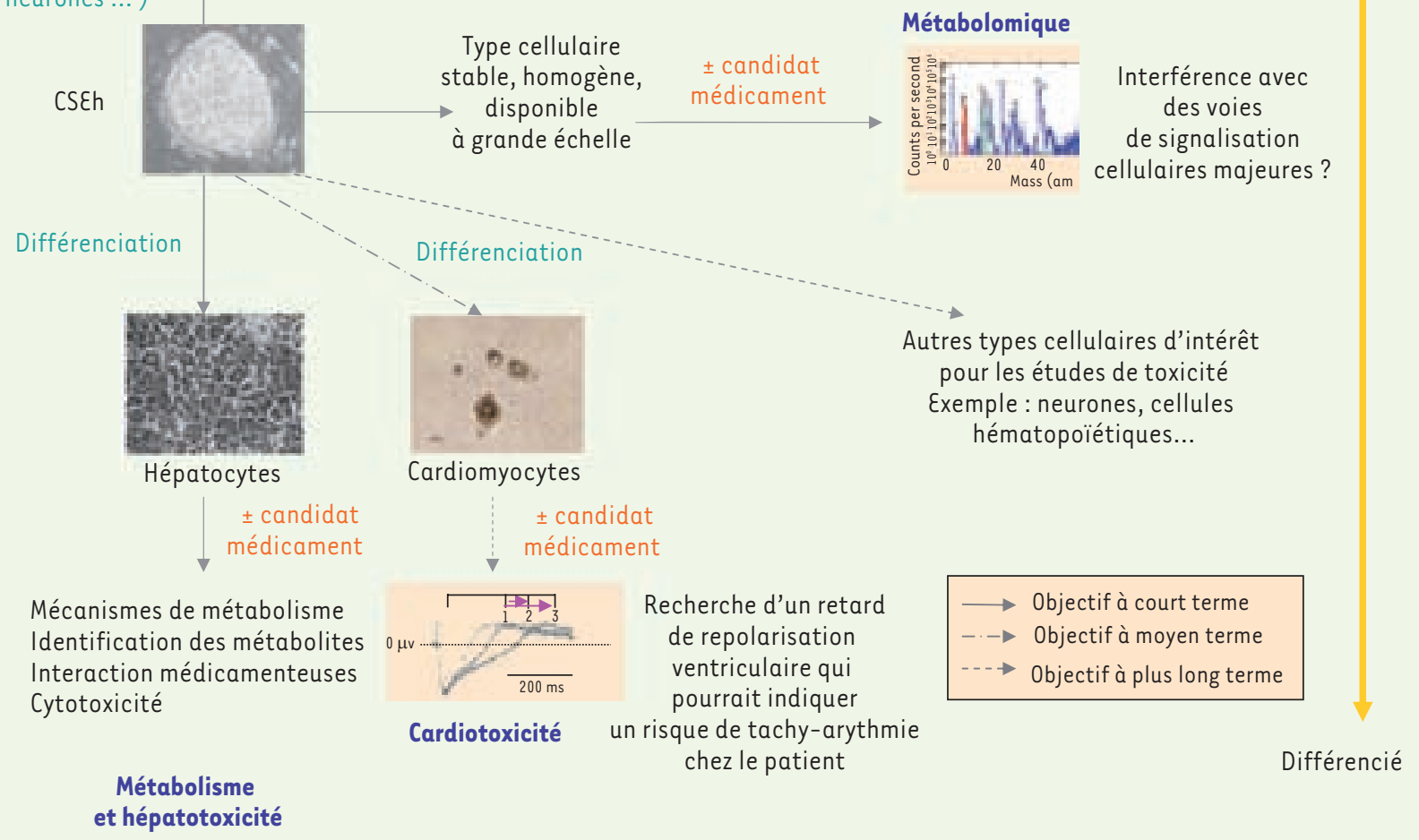

Figure 3. Cellules souches embryonnaires et toxicologie prédictive. 
de la solubilité des composés par ajout de groupements polaires). À titre d'exemple, ces cellules «hepatocyte-like » expriment le CyP3A4, isoforme du cytochrome P450 responsable de la métabolisation de plus de $50 \%$ des médicaments selon des réactions de phase I. Une étude récente portant sur de telles cellules issues de CSEh s'est intéressée à la glutathione transférase, enzyme de phase II [27]. Les auteurs ont montré que le profil des sous-unités qui la composaient, de même que son activité, étaient comparables à ceux observés dans des hépatocytes adultes humains. La machinerie enzymatique, vraisemblablement fonctionnelle, permet d'accéder à des modèles pertinents. Les cellules «hepatocyte-like » obtenues selon le protocole de Hay et al. [26] sont en effet capables de métaboliser le midazolam et le tolbutamide, alors que cette dernière molécule ne l'est pas par les cellules HepG2. Elles reproduisent en outre l'interaction médicamenteuse entre le midazolam et la rifampicine par induction enzymatique. La possibilité d'utiliser des lignées représentatives des variations génétiques naturelles présentes au sein de la population renforce l'intérêt de ces nouveaux modèles issus de CSEh dans cette étape de développement du médicament.

\section{De nouvelles approches pour revaloriser des médicaments écartés}

En parallèle de ces modèles spécialisés, une recherche se développe sur la conception de nouvelles approches de mesure de la toxicité des molécules. La société de biotechnologie américaine Stemina examine ainsi la possibilité d'exploiter les CSEh pour identifier, grâce à des études de «métabolomique » différentielle, les cibles moléculaires impliquées dans la toxicité de médicaments par ailleurs efficaces (Figure 3) [5]. Les chercheurs de Stemina espèrent ainsi pouvoir proposer des mesures spécifiquement adaptées à chaque cas, qui permettront de «sauver »certains médicaments actifs sur une pathologie mais écartés du fait d'effets indésirables.

\section{Innovations technologiques indispensables pour l'utilisation des CSEh dans l'industrie}

Les premiers modèles issus de CSEh seront vraisemblablement des modèles simples, unicellulaires. À l'heure actuelle, un défi important consiste à rendre des prototypes développés à l'échelle du laboratoire compatibles avec une utilisation standardisée à grande échelle, tout en tenant compte de l'évolution extrêmement rapide des connaissances dans le domaine des CSEh. Devant le nombre croissant de lignées, l'établissement de standards de caractérisation devient indispensable, afin de permettre leur comparaison et de mieux comprendre leurs différences (origine, méthode de dérivation, polymorphisme génétique, stabilité au cours des passages, potentiel de différenciation) [7]. Des bases de données telles que le « European Human Embryonic Stem Cell Registry » (http://www.hescreg.eu/) se développent afin de répondre à ces attentes. Concernant la culture des cellules, les protocoles d'amplification et de différenciation devront être standardisés. Cela implique d'éviter les systèmes de coculture, de travailler en milieu défini et avec des techniques de dissociation adaptées, tout en veillant à ne pas induire d'instabilité génétique [28]. Ces mêmes protocoles devront être compatibles avec un changement d'échelle, ce qui nécessite une automatisation des processus par des plates-formes robotiques [29] ou le recours à des bioréacteurs $[30,31]$ $(\rightarrow)$. Les protocoles de différenciation devront également être optimisés, de $(\rightarrow) \mathrm{m} / \mathrm{s} 2008$, $n^{\circ} 4$, p. 335 manière à tendre vers une population pure, homogène et la plus mature possible, les populations intermédiaires et terminales devant être caractérisées de manière approfondie. II faudra également identifier un progéniteur pouvant être stocké [32], et donc subir un cycle de congélation et de décongélation, sans que ses capacités de prolifération et de différenciation ne soient altérées.

Une fois ces défis relevés, la valeur ajoutée des modèles issus de CSEh pourra être pleinement évaluée. II sera alors possible d'envisager de passer à une seconde génération de modèles, plus perfectionnés, explorant la culture en 3 dimensions et l'association de plusieurs types cellulaires. Ces approches devraient tout d'abord favoriser l'accès à des stades de maturation plus avancés, même si leur origine embryonnaire ne permettra $a$ priori pas de les conduire jusqu'à un stade «adulte» dans des conditions de culture in vitro. Ces modèles élaborés, qui seront destinés non plus à une utilisation à grande mais à petite échelle, devraient permettre de se rapprocher de structures tissulaires et ainsi de générer des systèmes à forte prédictivité, notamment pour l'étude de certaines structures telles que les barrières hémato-encéphalique ou hématoplacentaire.

\section{Conclusions}

Alors que le coût de développement d'un médicament, qui s'élevait à 900 millions de dollars en 2004 [33], est toujours plus important, le nombre de nouvelles molécules autorisées sur le marché ne cesse de diminuer. À titre d'exemple, 36 nouveaux médicaments ont été approuvés par la FDA en 2004 contre 20 seulement en 2005 [7]. Limiter l'attrition lors des essais chez l'animal et chez le patient par le développement de nouveaux modèles in vitro plus prédictifs devrait permettre de réduire significativement les investissements. $\varepsilon$ n effet, en $2001,30 \%$ des médicaments entrant en essais cliniques étaient retirés pour un manque d'efficacité et $30 \%$ suite à un défaut de sécurité [33]. L'industrie pharmaceutique n'est pas la seule concernée. La directive européenne REACH (registration, evaluation, authorisation and restriction of chemicals) sur l'évaluation de la dangerosité des substances chimiques, 
ainsi que l'interdiction de l'expérimentation animale dans l'industrie cosmétique confirment cette urgente nécessité de développer de nouveaux modèles in vitro, à la fois robustes et économiques [34]. Les cellules souches embryonnaires humaines et peut-être demain les iPS pourraient y contribuer. $\diamond$

\section{SUMMARY}

\section{Embryonic stem cells in pharmacology}

Because of their self-renewal and pluripotency properties, human embryonic stem cells (hES) receive a marked attention from scientists and clinicians for regenerative medicine. The most recent application of hES cells may however reside in their use as a tool in drug development. The currently available cellular models for preclinical testing consist in primary and immortalized cells that display limitations in terms of available amount and likeliness to their in vivo counterparts, respectively. hES cells have the potential to revolutionize drug discovery by providing a physiological model for any human cell type in the desired amount for the earliest steps of drug development, notably for pharmacological, metabolic and toxicity evaluation. This new generation of model may contribute to reduce, refine or replace animal testing and decrease drug attrition. $\diamond$

\section{REMERCIEMENTS}

Nous remercions Marie-Claude Kilhoffer pour l'aide apportée au début de cette étude.

\section{ILLUSTRATIONS}

La Figure 1 est adaptée de [35]. Dans la Figure 3, la photo des hépatocytes provient de la figure 1B de [26]. La photo des cardiomyocytes provient de la figure 5 de [7]. L'illustration du potentiel d'action ventriculaire provient de [36]. L'illustration du métabolome provient de la diapositive 13 de [37].

\section{RÉFÉRENCES}

1. Thomson JA, Itskovitz-Eldor J, Shapiro SS, et al. Embryonic stem cell lines derived from human blastocysts. Science $1998 ; 282$ : 1145-7.

2. Smith AG. Embryo-derived stem cells: of mice and men. Annu Rev Cell Dev Biol 2001 ; $17: 435-62$.

3. Gorba T, Allsopp TE. Pharmacological potential of embryonic stem cells. Pharmacol Res 2003 ; 47: 269-78.

4. McNeish J. Embryonic stem cells in drug discovery. Nat Rev Drug Discov $2004 ; 3: 70-80$.

5. Cezar GG. Can human embryonic stem cells contribute to the discovery of safer and more effective drugs? Curr Opin Chem Biol 2007 ; $11: 405-9$.

6. McNeish JD. Stem cells as screening tools in drug discovery. Curr Opin Pharmacol 2007 ; $7: 515-20$.

7. Sartipy $P$, Björquist $P$, Strehl R, Hyllner J. The application of human embryonic stem cell technologies to drug discovery. Drug Discovery Today $2007 ; 12: 688-99$.

8. Ameen C, Strehl R, Bjorquist P, et al. Human embryonic stem cells: current technologies and emerging industrial applications. Crit Rev Oncol Hematol 2008 ; 65 : 54-80.

9. Wilson PG, Cherry JJ, Schwamberger S, et al. An SMA project report: neural cell-based assays derived from human embryonic stem cells. Stem Cells Dev 2007 ; 16 : 1027-41.

10. Coulombel L, De Vos J, Puceat M. FISH-ESC : premier colloque international sur la recherche dédiée aux cellules souches embryonnaires humaines. Med Sci (Paris) 2008 ; $24: 419-26$.

11. Pickering SJ, Braude PR, Patel M, et al. Preimplantation genetic diagnosis as a novel source of embryos for stem cell research. Reprod Biomed (Online) $2003 ; 7: 353-64$.

12. Verlinsky $Y$, Strelchenko N, Kukharenko V, et al. Human embryonic stem cell lines with genetic disorders. Reprod Biomed (Online) $2005 ; 10: 105-10$.
13. Mateizel I, De Temmerman N, Ullmann U, et al. Derivation of human embryonic stem cell lines from embryos obtained after IVF and after PGD for monogenic disorders. Hum Reprod 2006 ; 21 : 503-11.

14. Eiges R, Urbach A, Malcov M, et al. Developmental study of fragile $X$ syndrome using human embryonic stem cells derived from preimplantation genetically diagnosed embryos. Cell Stem Cell 2007 ; $1: 568-77$.

15. Takahashi K, Tanabe K, Ohnuki M, et al. Induction of pluripotent stem cells from adult human fibroblasts by defined factors. Cell 2007 ; $131: 861-72$.

16. $\mathrm{Yu}_{\mathrm{u}}$ J, Vodyanik MA, Smuga-0tto K, et al. Induced pluripotent stem cell lines derived from human somatic cells. Science 2007 ; 318 : 1917-20.

17. Coulombel L. Cellules iPS humaines : déjà ! Med Sci (Paris) 2008 ; $24: 102-4$.

18. Rigamonti $D$, Bolognini $D$, Mutti $C$, et al. Loss of huntingtin function complemented by small molecules acting as repressor element 1 /neuron restrictive silencer element silencer modulators. J Biol Chem 2007 ; $282: 24554-62$.

19. Leone S, Mutti C, Kazantsev A, et al. SAR and QSAR study on 2aminothiazole derivatives, modulators of transcriptional repression in Huntington's disease. Bioorg Med Chem 2008 ; 16 : 5695-703.

20. Spielmann H, Pohl I, Doering B, et al. The embryonic stem cell test, an in vitro embryotoxicity test using two permanent mouse cell lines: 3T3 fibroblasts and embryonic stem cells. In Vitro Toxicology 1997 ; 199-227

21. Genschow $\varepsilon$, Spielmann H, Scholz G, et al. Validation of the embryonic stem cell test in the international ECVAM validation study on three in vitro embryotoxicity tests. Altern Lab Anim 2004 ; 32 : 209-44.

22. Adler S, Pellizzer C, Hareng L, et al. First steps in establishing a developmental toxicity test method based on human embryonic stem cells. Toxicol In Vitro $2008 ; 22: 200-11$.

23. Hareng L, Pellizzer C, Bremer $S$, et al. The integrated project ReProTect: a novel approach in reproductive toxicity hazard assessment. Reprod Toxicol $2005 ; 20: 441-52$.

24. Wilkening $S$, Stahl F, Bader A. Comparison of primary human hepatocytes and hepatoma cell line Hepg2 with regard to their biotransformation properties. Drug Metab Dispos 2003; 31 : 1035-42.

25. Agarwal S, Holton KL, Lanza R. Efficient Differentiation of functional hepatocytes from human embryonic stem cells. Stem Cells 2008 (online).

26. Hay DC, Zhao D, Fletcher J, et al. Efficient differentiation of hepatocytes from human embryonic stem cells exhibiting markers recapitulating liver development in vivo. Stem Cells 2008; $26: 894-902$.

27. SoderdahI T, Kuppers-Munther B, Heins N, et al. Glutathione transferases in hepatocyte-like cells derived from human embryonic stem cells. Toxicol In Vitro $2007 ; 21: 929-37$.

28. Pouton CW, and Haynes JM. Embryonic stem cells as a source of models for drug discovery. Nat Rev Drug Discov 2007 ; 6: 605-16.

29. Terstegge S, Laufenberg I, Pochert J, et al. Automated maintenance of embryonic stem cell cultures. Biotechnol Bioeng $2007 ; 96: 195-201$.

30. Thomson H. Bioprocessing of embryonic stem cells for drug discovery. Trends Biotechnol $2007 ; 25: 224-30$.

31. Peschanski M. Cellules souches: I'heure venue du changement d'échelle. Med Sci (Paris) $2008 ; 24: 335-8$.

32. Pouton CW, and Haynes JM. Pharmaceutical applications of embryonic stem cells. Adv Drug Deliv Rev 2005 ; 57 : 1918-34.

33. Kola I, Landis J. Can the pharmaceutical industry reduce attrition rates? Nat Rev Drug Discov 2004 ; 3 : 711-5.

34. Bremer S, Pellizzer C, Hoffmann S, et al. The development of new concepts for assessing reproductive toxicity applicable to large scale toxicological programmes. Curr Pharm Des 2007 ; 13 : 3047-58.

35. Trounson A. The production and directed differentiation of human embryonic stem cells. Endocrinol Rev 2006 ; 27 : 208-19.

36. Reppel M, Pillekamp, Brockmeier K, et al. The electrocardiogram of human embryonic stem cell-derived cardiomyocytes. J Electrocardiol 2005 ; 38 (suppl 4) : 166-70.

37. Stemina presents at tri-molecular conference. Présentation téléchargeable sur le site web de la société Stemina. http://www.stemina.com/

\section{TIRÉS À PART}

D. Laustriat et

M. Peschanski 